\title{
ADOÇÃO TECNOLÓGICA E SEUS CONDICIONANTES: O CASO DA BANANICULTURA NO AGROPOLO CARIRI-CE ${ }^{1}$
}

\author{
Maria Aparecida Silva Oliveira ${ }^{2}$ \\ Ahmad Saeed Khan ${ }^{3}$ \\ Patrícia Verônica Pinheiro Sales Lima
}

\begin{abstract}
Resumo - A utilização de tecnologia na agricultura a torna produtiva e promissora, contribuindo para o seu desenvolvimento. Os investimentos na agricultura realizados pelo governo do Ceará têm-se concentrado principalmente na agricultura irrigada, através dos agropolos, predominando a fruticultura, sendo a banana uma das principais culturas. A bananicultura desempenha importante papel para a agricultura cearense, entretanto é caracterizada, geralmente, pela baixa produtividade, baixo nível tecnológico e grandes perdas na produção. Os objetivos deste trabalho foram mensurar o nível tecnológico da bananicultura irrigada no Agropolo Cariri e verificar que fatores condicionam a adoção tecnológica. O índice calculado indicou que o nível tecnológico adotado na bananicultura se classifica como bom. As tecnologias de irrigação e fitossanidade têm nível de adoção classificado como ótimo e as de mudas, adubação e tratos culturais, como bom. Os níveis das tecnologias de colheita e pós-colheita são classificados como regulares e os da gestão, como insuficientes. As variáveis socioeconômicas assistência técnica, bananicultura como atividade principal, crédito, escolaridade, idade, posse da terra, renda e local de residência contribuem positivamente para aumentar a probabilidade de o produtor adotar tecnologia adequada na bananicultura.

Palavras-chave: Adoção tecnológica, bananicultura, Agropolo Cariri.
\end{abstract}

1 Recebido em 01/07/2005 Aceito em 02/09/2005

2 Economista, mestre em Economia Rural, doutoranda em Economia Aplicada da Universidade Federal de Viçosa Endereço: Rua Jose Ubaldo de Paiva, 39 ap. 304. Bairro de Ramos - 36570-000. Viçosa - MG. Tel: (31) 3885-1150

3 Professor titular do Departamento de Economia Agrícola - UFC. Bolsista do CNPq. E-mail: saeed@ ufc.br

4 Professora do Departamento de Economia Agrícola - UFC. E-mail: <p.sales.lima@ uol.com.br>. 


\section{Introdução}

O governo do Estado do Ceará implantou, no final da década de 1990, o Programa Cearense de Agricultura Irrigada, com o intuito de mudar as condições de baixa produtividade da agricultura cearense e inseri-la, de forma competitiva, em mercados estratégicos, através do uso da irrigação, nos agropólos ${ }^{5}$ (Secretaria de Agricultura Irrigada - SEAGRI, 1999). Para que os agropólos possam desempenhar esses papéis no setor agrícola do estado, é necessário que um nível de tecnologia adequado seja adotado.

A tecnologia apresenta-se intimamente ligada a variações da produtividade e ocasiona outras mudanças no processo de produção, pois proporciona menor grau de dependência da agricultura aos fatores naturais. As inovações nas áreas da Biologia, Mecânica e Química têm permitido maior controle sobre o processo produtivo na agricultura, possibilitando suprir as deficiências de solo, água e clima, que possam inviabilizar a produção.

Como enfatizou Elias (1996), a utilização de tecnologia na agricultura a torna um empreendimento tão promissor quanto os empreendimentos realizados nos demais setores da economia, com a capacidade de proporcionar alta lucratividade e maior competitividade, além de valorar os capitais nela investidos.

A adoção de tecnologias é uma variável qualitativa e condicionada tanto por características econômicas, culturais e sociais quanto também subjetivas dos produtores. Essas características influenciam a percepção de mundo do agricultor e a forma como ele enxerga os problemas e encontra formas de solucioná-los (Mesquita, 1998).

Alguns autores já objetivaram mensurar o nível de adoção tecnológica no setor agrícola e verificar seus aspectos condicionantes. Santos (1977) identificou os fatores que influenciam a adoção de práticas agropecuárias no Estado de Minas Gerais e especificou o nível tecnológico adotado por

Os agropolos são: Metropolitano, Jaguaribe, Ibiapaba, Baixo Acaraú, Centro-Sul, Cariri e Sertão Central. 
um índice determinado pelo número de práticas empregadas. Para analisar os fatores determinantes da adoção de tecnologia na cajucultura cearense, Carbajal (1991) calculou o nível tecnológico, atribuindo escores às tecnologias utilizadas, e os ponderou pelas suas importâncias relativas. Silva e Carvalho (2002) também usaram o método da contagem para especificar o nível tecnológico e avaliar os fatores que determinam a sua adoção em regiões paulistas.

O objetivo deste trabalho foi mensurar o nível tecnológico da bananicultura adotado nos agropolos cearenses, bem como verificar os seus fatores condicionantes. A escolha da bananicultura para a análise deve-se a três motivos. Primeiro, a fruticultura é a principal atividade desenvolvida nos agropolos, e a bananicultura apresenta-se como uma das principais culturas em todos os agropolos do Ceará. Além disso, a banana serve como alimento básico para a população de baixa renda, sendo preferida pelo preço popular e por possuir alto valor energético, o que lhe confere importância social (Souza, 2002). No entanto, a banana está entre as frutas mais comercializadas no mundo, tendo boa aceitação nos principais mercados importadores de frutas frescas, possuindo, portanto, grande potencial exportador (Faveret Filho et al., 1999).

Para se ter um nível tecnológico que reflita com maior fidelidade como a bananicultura é praticada no estado, escolheu-se o Município de Mauriti, no Agropolo Cariri, para a realização da pesquisa. Nesse município, na época em que a pesquisa foi realizada, não existiam grandes produtores que adotassem tecnologia com grandes graus de diferenciação, garantindo que o índice a ser calculado não seja superestimado, obtendo-se, assim, boa representatividade da tecnologia da bananicultura do agropolo e do Ceará, dado que a predominância, nesses locais, é de pequenos produtores.

De forma adicional, consideraram-se na formulação do índice tecnológico não somente as tecnologias adotadas, como realizado nos trabalhos anteriormente citados, mas também as técnicas que as compõem. Isso porque as técnicas possuem diversificados graus de eficiência, relevantes na percepção adequada do nível tecnológico, e, quando não consideradas, 
pode-se obter uma visão equivocada sobre o nível de adoção das tecnologias.

As informações sobre o nível tecnológico com que a bananicultura está sendo praticada e que fatores condicionam sua adoção podem subsidiar os tomadores de decisão na formulação, ou reformulação, de soluções para problemas relacionados à cultura da banana no Agropolo Cariri e nos demais agropolos produtores do estado.

Além desta introdução, o presente artigo contém a metodologia com o índice tecnológico utilizado para mensurar o nível tecnológico, o modelo utilizado na determinação dos fatores condicionantes da sua adoção e a fonte e operacionalização dos dados. Em seguida são expostos os resultados e discussão e as conclusões do trabalho.

\section{Metodologia}

\subsection{Mensuração do nível tecnológico}

As variáveis consideradas na composição do índice tecnológico foram: mudas, irrigação, adubação, tratos culturais, fitossanidade, colheita, póscolheita e gestão.

Para cada propriedade pesquisada e cada uma das tecnologias referentes às variáveis especificadas foi determinado um índice tecnológico, dado por:

$$
I_{b j}=\sum_{r=g}^{k} \frac{m_{r}}{s_{b}}
$$

em que b é a $b$-ésima tecnologia $(\mathrm{b}=1,2, \ldots, \mathrm{v})$; r é o $r$-ésimo segmento referente à tecnologia $b[\mathrm{r}=1,2, \ldots, \mathrm{k}]$, ou seja, em $\mathrm{r}$ está o número de $\mathrm{k}$ técnicas pertencentes a cada tecnologia $\mathrm{b}$; $\mathrm{m}_{\mathrm{r}}$ é o valor do escore de uma variável ou técnica da tecnologia $b ;\left[\mathrm{g}_{\mathrm{b}} ; \mathrm{k}_{\mathrm{b}}\right]$ são as variáveis de um segmento $r$ referentes à tecnologia $b ; \mathrm{j}$ é o j-ésimo produtor $(\mathrm{j}=1,2, \ldots, \mathrm{n})$; 
$I_{b j}$ é o índice da tecnologia $b$, da propriedade $j$; e $\frac{m_{r}}{s_{b}}$ é o peso de uma variável ou técnica na constituição do índice da tecnologia $b$, sendo $s_{b}=$ Max Sm e, dessa forma, $0 \leq I_{b j} \leq 1$, ou seja, $s_{b}$ ocorreria quando o nível tecnológico das variáveis citadas fosse o mais elevado e, portanto, $I_{b j}$ seria igual a 1 .

O índice tecnológico para cada propriedade $\left(I_{G}\right)$ foi calculado como a seguir:

$$
I_{G}=\frac{1}{v} \sum_{b=1}^{v} \sum_{r=g}^{k} \frac{m_{r}}{s_{b}}
$$

$\mathrm{O}$ índice tecnológico médio de todas as propriedades $\left(\mathrm{I}_{\mathrm{Gn}}\right)$ pesquisadas foi dado por:

$$
I_{G n}=\frac{1}{n} \sum_{j=1}^{n} I_{G}
$$

Para melhor realização da análise e comparação dos dados, o índice tecnológico do produtor foi dividido em quartis, estabelecendo padrões de níveis tecnológicos, como especificado a seguir. O estabelecimento dos padrões nesses intervalos foi feito de acordo com a metodologia sugerida por Miranda (2001).

Se $0,75 \leq \mathrm{I}_{\mathrm{Gn}}<1,00$, o produtor $j$ tem o padrão I de tecnologia;

Se $0,50 \leq \mathrm{I}_{\mathrm{Gn}}<0,75$, o produtor $j$ tem o padrão II de tecnologia;

Se $0,25 \leq \mathrm{I}_{\mathrm{Gn}}<0,50$, o produtor $j$ tem o padrão III de tecnologia; e

Se $0,00 \leq \mathrm{I}_{\mathrm{Gn}}<0,25$, o produtor $j$ tem o padrão IV de tecnologia. 
O padrão tecnológico I classifica os produtores que utilizam $75 \%$ ou mais da tecnologia recomendada para mudas, adubação, irrigação, tratos culturais, fitossanidade, colheita, pós-colheita e gestão na produção de banana, podendo ser considerado como ótimo o padrão de tecnologia adotado. Da mesma forma, o padrão II classifica a produção de banana que utiliza entre 50 e $75 \%$ da tecnologia recomendada, sendo este considerado um bom padrão tecnológico. De igual modo, o padrão III está relacionado à adoção de um padrão de tecnologia regular, e o padrão IV refere-se à adoção de um padrão insuficiente de tecnologia para a produção de banana, de acordo com os intervalos percentuais de adoção estabelecidos.

\subsection{Relação entre uso de tecnologia e características} socioeconômicas dos produtores

A tomada de decisão sobre a adoção de certa tecnologia conduz a uma das respostas: adotar ou não adotar. Dessa forma, essa variável é dicotômica ou binária, de natureza qualitativa e, como enfatizado, é dependente de outras variáveis. Segundo Lima (1996), para fenômenos dessa natureza a resposta binária $\left(\mathrm{Y}_{\mathrm{i}}\right)$ é função de um índice latente $\left(\mathrm{I}_{\mathrm{i}}\right)$, que varia de um mínimo a um máximo passando por um nível-limite (I*), que determina uma mudança de qualidade na resposta de um indivíduo, ou seja:

$\mathrm{Y}=1$, quando $\mathrm{I}_{\mathrm{i}}>\mathrm{I}^{*}$

$\mathrm{Y}=0$, quando $\mathrm{I}_{\mathrm{i}} \leq \mathrm{I}^{*}$

em que $i$ representa a $i$-ésima observação.

Neste estudo, a variável Y assume o valor 1 quando a decisão for adotar a tecnologia e zero para o caso contrário.

A resposta binária (Yi) é uma função da variável latente, conforme a equação a seguir: 
$Y_{i}=f\left(I_{i}\right)$

A variável latente, por sua vez, é função dos $k$ atributos do indivíduo $\left(\mathrm{X}_{1}\right.$, $\mathrm{X}_{2}, \ldots \mathrm{X}_{\mathrm{k}}$ ), podendo a função (4) ser expressa como a seguir:

$$
Y_{i}=F\left(X_{i}^{\prime} \beta\right)
$$

em que $\beta$ é o vetor de parâmetros a serem estimados, e $X_{i}$ é a matriz das variáveis socioeconômicas dos produtores.

A probabilidade de ocorrer ou não um evento é dada por:

$$
\begin{aligned}
& P(Y=1)=P\left(I>I^{*}\right)=P_{i}=F\left(X_{i}{ }^{\prime} \beta\right) \\
& P(Y=0)=P\left(I \leq I^{*}\right)=1-P_{i}=1-F\left(X_{i}{ }^{\prime} \beta\right)
\end{aligned}
$$

Respectivamente, sendo $\mathrm{P}_{\mathrm{i}}$ a probabilidade de ocorrência da i-ésima observação.

As formas funcionais utilizadas para esse tipo de modelo probabilístico são: Modelo de Probabilidade Linear (MPL), Logit e Probit. O primeiro é estimado pelo método usual de regressão linear, através dos Mínimos Quadrados Ordinários. O MPL traz alguns problemas ${ }^{6}$ de violação dos pressupostos, como: não-normalidade das perturbações estocásticas, variância heteroscedástica das perturbações, estima probabilidade fora do intervalo 0 e 1 e tem valor questionável de $\mathrm{R}^{2}$ como medida do grau de ajuste.

Como o principal problema do MPL reside na estimação de probabilidades não situadas no intervalo de 0 a 1 , deve-se utilizar uma função $F$ que atenda a essa condição. Os modelos Logit e Probit são baseados em funções distribuição logística cumulativa e distributiva normal cumulativa, respectivamente, que estimam probabilidades dentro do intervalo esperado. As formas funcionais desses modelos são dadas a seguir:

\footnotetext{
6 Para maiores detalhes, ver Gujarati (2000).
} 
Logit: $F\left(X_{i}^{\prime}, \beta\right)=L\left(X_{i}^{\prime} \beta\right)=\frac{1}{1+e^{X_{i}^{\prime} \beta}}$

Probit: $F\left(X_{i}^{\prime}, \beta\right)=\phi\left(X_{i}^{\prime} \beta\right)=\int_{-\infty}^{X_{i}{ }^{\prime} \beta} \frac{1}{\sqrt{2 \pi}} e^{-t^{2} / 2} d t$

em que $e$ é a base do logaritmo natural e p, uma constante com valor aproximado de 3,1416 .

A variação da variável explicada (a probabilidade) dada por uma variação na variável independente é dada pelo coeficiente estimado somente no MPL. Nos modelos Logit e Probit, essa variação é chamada de Efeito Marginal e dada pela inclinação da curva de distribuição de probabilidade dos referidos modelos. Assim, de acordo com Lima (1996), os efeitos marginais são calculados por derivadas parciais, como mostrado a seguir:

Modelo Probit: $\frac{\partial}{\partial x_{i k}} \phi\left(X_{i}^{\prime} \beta\right)=\phi\left(X_{i}^{\prime} \beta\right) \cdot \beta_{k}$

Modelo Logit: $\frac{\partial}{\partial x_{i k}} L\left(X_{i}^{\prime} \beta\right)=\frac{e^{X_{i}^{\prime} \beta}}{\left(1+e^{X_{i}^{\prime} \beta}\right)^{2}} \cdot \beta_{k}$

Para verificar se a contribuição das variáveis independentes é significativa, é calculada a Razão de Máxima Verossimilhança ou Estatística LR. Esse teste é semelhante ao teste "F" de Snedecor aplicado aos modelos estimados com métodos dos Mínimos Quadrados Ordinários, isto é, sua hipótese de nulidade é que as variáveis independentes em conjunto sejam iguais a zero e a hipótese alternativa é o caso contrário.

De acordo com Greene (1997), a estatística LR é calculada como indicada na equação (12) e segue distribuição qui-quadrada $\left(\chi^{2}\right)$, com q graus de liberdade, sendo q o número de variáveis independentes. 


$$
L R=-2\left(\ln L_{R}-\ln L\right)
$$

em que $L_{R}$ e $L$ são os valores das funções de máxima verossimilhança estimadas com restrição (vetor de coeficientes restrito a zero, isto é, $\beta=0$ ) e sem restrição (vetor de coeficientes irrestrito, isto é, $\beta \neq 0$ ).

Como explicitado, o coeficiente de determinação $\mathrm{R}^{2}$ não é uma medida de ajuste confiável para modelos de resposta binária. Madalla (1992) sugeriu algumas formas opcionais para mensuração do grau de ajuste. Neste trabalho foi utilizado o coeficiente de McFadden $\mathrm{R}^{2}$, calculado conforme a seguir:

$$
\text { McFadden } R^{2}=1-\frac{\log L}{\log L_{R}}
$$

A heteroscedasticidade, comum em trabalhos que envolvem dados microeconômicos, é testada pela estatística do Multiplicador de Lagrange. De acordo com Santos (2000), para realizar o teste, utiliza-se a seguinte expressão:

$$
\operatorname{Var}\left(e_{i}\right)=\exp (z \gamma)^{2}
$$

em que Var( $\left.\mathrm{e}_{\mathrm{i}}\right)$ é a variância do termo de perturbação estocástica; exp é $e$ (base do logaritmo natural) elevado à expressão entre parênteses; $\mathrm{z}$ é um vetor de variáveis independentes que representa a fonte de heterocedasticidade; e $y$ é o vetor de coeficientes.

Para detectar a heterocedasticidade, deve-se testar a significância de $y$ pelo teste de verossimilhança. A hipótese de nulidade é que $y=0$, e, assim, a variância é homocedástica. Na hipótese alternativa $y \neq 0$, a variância é heterocedástica. 


\subsection{Fonte dos dados e operacionalização das variáveis}

Os dados utilizados foram primários, colhidos diretamente através da aplicação de questionário a uma amostra dos produtores de banana irrigada do Município de Mauriti, no agropolo da região do Cariri Cearense. A coleta de dados ocorreu em outubro de 2002.

Para formulação do índice tecnológico da produção de banana foram consideradas não só as tecnologias, mas também as técnicas que as compõem. Para cada variável ou técnica que compõe determinada tecnologia foi atribuído um escore, de acordo com a sua eficiência. Para a tecnologia de mudas foram consideradas as técnicas de preparo e origem da muda. $\mathrm{Na}$ tecnologia de irrigação foram atribuídos escores crescentes para as técnicas de irrigação mais adequadas para a cultura da bananeira. Na adubação foi observada a utilização das técnicas de adubação de implantação e manutenção. Para a tecnologia de tratos culturais foram consideradas as utilizações ou não das principais técnicas recomendadas ${ }^{7}$ para a cultura. De igual forma, na tecnologia de fitossanidade foram verificadas as utilizações de técnicas de combate ou controle das principais pragas e doenças ${ }^{8}$ que atacam os bananais. Para a tecnologia de colheita foram consideradas as técnicas de ponto e forma de colheita e para a pós-colheita, as técnicas de lavagem, seleção e climatização dos frutos. Na tecnologia de gestão foi verificada a existência de treinamento da mão-de-obra e utilização de informática para fins organizacionais, comerciais e mercadológicos.

As variáveis socioeconômicas posse da terra, acesso ao crédito e assistência técnica e bananicultura como atividade principal, explicativas da adoção de tecnologia, foram mensuradas de forma binária, ou seja, foi atribuído o valor 1 quando ocorriam na propriedade pesquisada, e zero para o caso contrário. Para a variável residência também foi

\footnotetext{
Os tratos culturais recomendados são: capina, desbrota ou desbaste, escoramento, ensacamento do cacho, desfolha e cobertura morta.

8 As principais pragas que atacam bananais são: moleque, tripes e traça da bananeira. E as principais doenças são: sigatoka-amarela, mal-do-panamá e nematóide.
} 
construída uma dummy, sendo o valor 1 atribuído quando o produtor mora na propriedade e zero quando isso não acontece. Para a variável renda total foi considerada a renda familiar do agricultor, incluindo a renda agrícola e não-agrícola. Para a escolaridade foi construída uma variável discreta com os valores de zero a 3 para os casos de produtores analfabetos ou semi-analfabetos, que cursaram os ensinos fundamental, médio e superior, respectivamente. A disponibilidade de mão-de-obra foi mensurada pela equivalência-homem da mão-de-obra familiar disponível. Para a variável idade foram considerados os anos de vida do produtor.

\section{Resultados e discussão}

\subsection{Nível Tecnológico}

$\mathrm{O}$ índice $\mathrm{I}_{\mathrm{Gn}}$, ou índice tecnológico geral, encontrado para a amostra pesquisada foi igual a 0,52 , indicando que a produção de banana do Município de Mauriti encontra-se no padrão II de tecnologia (Tabela 1). A razão disso está na não-utilização ou pouco emprego de algumas das técnicas mais eficientes das tecnologias que compõem o processo produtivo.

Os resultados obtidos em relação aos padrões das tecnologias utilizadas são característicos dos pólos de fruticultura irrigada implantados no Nordeste brasileiro, pois, de acordo com Cordeiro (2000), embora esses pólos sejam uma exceção às demais regiões produtoras de banana do país com baixa tecnificação, eles apresentam melhor produtividade, em virtude do uso da irrigação, mas, no entanto, deixam muito a desejar em relação ao manejo e tratos culturais dispensados à cultura e ao tratamento de colheita e pós-colheita. 
Tabela 1 - Padrões tecnológicos adotados na bananicultura de Mauriti, CE

\begin{tabular}{c}
\hline Tecnologias \\
\hline Irrigação \\
Fitossanidade \\
\hline Mudas \\
Adubação \\
Tratos culturais \\
\hline Colheita \\
Pós-colheita \\
\hline Gestão \\
\hline $\mathrm{I}_{\mathrm{Gn}}$ \\
\hline
\end{tabular}

Fonte: Dados da pesquisa.

A principal razão de a irrigação estar no padrão de tecnologia mais elevado é o fato de o município estar inserido em um dos agropolos desenvolvidos pelo governo do Estado do Ceará, através da SEAGRI, onde a irrigação é orientada e estimulada para os agricultores. Isso ressalta a importância de políticas de desenvolvimento agrícola para a elevação do nível tecnológico adotado.

Em média, $80 \%$ das propriedades pesquisadas não sofrem infestação das principais pragas e doenças que atacam os bananais. Entre as propriedades que enfrentam esses problemas, em média $82 \%$ praticam alguma forma de combate ou controle. Disso resulta o fato de a fitossanidade encontrar-se no padrão I de tecnologia.

Embora a prática de fazer limpeza de mudas antes do plantio seja comum entre os produtores, mais da metade deles utiliza mudas retiradas diretamente do bananal. Isso faz com que a tecnologia de mudas se classifique no padrão II, pois esse tipo de muda é de qualidade inferior e seu uso ocasiona produção aquém da capacidade da bananeira, produção tardia e colheitas irregulares, além de elevarem os custos de produção, por apresentarem problemas fitossanitários.

A adubação da implantação foi realizada pela totalidade dos produtores. Entretanto, a adubação de manutenção não é realizada da forma mais 
eficiente, que seria a realizada por estágio de desenvolvimento da planta, fazendo a tecnologia de adubação classificar-se no padrão II. A nãoutilização dos nutrientes necessários ao desenvolvimento da planta, na maioria das vezes, ocorre devido ao desconhecimento do solo e da exigência nutricional da cultura por parte do produtor, levando-o à prática da adubação inadequada, o que afeta significativamente o desenvolvimento e a produtividade da bananeira.

A tecnologia de tratos culturais classificou-se no padrão II. Isso ocorreu, pois, das práticas recomendadas, $66 \%$ em média foram utilizadas nos bananais do município, indicando não haver grande negligência quanto aos tratos culturais por parte dos produtores.

A colheita e pós-colheita apresentaram padrão III de tecnologia devido à forma arcaica como os bananicultores colhem e embalam o produto. A falta de proteção para os cachos e de equipamentos adequados para o transporte deixa os frutos vulneráveis a atritos, machucando-se e prejudicando o produtor no momento da comercialização.

A gestão obteve o mais baixo padrão de tecnologia. Isso se deve, principalmente, à quase inexistência de treinamento da mão-de-obra utilizada na produção de banana. É importante o adequado treinamento da mão-de-obra, principalmente para colheita e pós-colheita, para evitar danos aos frutos. A falta de treinamento da mão-de-obra pode comprometer as demais tecnologias utilizadas, em razão do uso ou prática incorreta. Isso pode levar a uma redução do nível tecnológico geral, uma vez que o fator mão-de-obra tem influência sobre todo o processo produtivo da banana.

\subsection{Fatores condicionantes da adoção de tecnologia}

Estão apresentados nesta seção os resultados da estimação do modelo de variável dependente dicotômica. Para utilizar o índice tecnológico $\mathrm{I}_{\mathrm{Gn}}$ na forma dicotômica, considerou-se o valor 1 para os produtores com 
níveis tecnológicos pertencentes aos padrões I e II e 0, para os pertencentes aos padrões III e IV, de acordo com os intervalos estabelecidos.

A equação foi estimada através dos modelos Logit e Probit, descritos anteriormente. Como para a equação estimada houve maior número de variáveis estatisticamente significativas no modelo Probit em relação ao modelo Logit, o primeiro foi o escolhido.

Através da equação estimada foram analisadas as variáveis que influenciam a decisão de o produtor adotar um nível tecnológico adequado ou próximo do adequado 9 . Na Tabela 2, mostram-se esses resultados, já testados, e rejeitada a hipótese de heterocedasticidade.

O McFadden $\mathrm{R}^{2}$ encontrado foi igual a 0,6403. Esse valor indica que há um ajustamento aceitável no modelo, uma vez que, quanto mais próximo de 1 o valor do índice, melhor o ajustamento. A razão de máxima verossimilhaça (estatística LR) mostrou-se significativa no nível de 1\%, indicando que os coeficientes estimados das variáveis explicativas, em conjunto, têm influência sobre a variável explicada.

A variável Assistência Técnica se mostrou significante no nível de 5\%. O sinal positivo revelou que o produtor que tem assistência técnica tem maior possibilidade de adotar níveis tecnológicos adequados na produção de banana. No trabalho de Cavalcanti (1998), a falta de assistência técnica foi detectada significativa como uma das principais causas do não-emprego de tecnologia na agricultura.

Grande parte dos bananicultores de Mauriti produzia banana há menos de 5 anos. Nessa fase, em que o produtor não tem experiência com a cultura e está adquirindo conhecimentos sobre ela, a assistência técnica é de grande relevância, podendo-se atribuir a isso, entre outros fatos, sua significância sobre a probabilidade de adoção de tecnologia para esses produtores.

Foram considerados níveis adequados os índices tecnológicos pertencentes aos padrões I e II. 
Tabela 2 - Regressão dos condicionantes da probabilidade de adoção de tecnologia dos produtores de banana de Mauriti, usando o modelo Probit

\begin{tabular}{lr}
\hline \multicolumn{1}{c}{ Variáveis } & Coe \\
\hline Constante & -1 \\
Assistência técnica & 2 \\
Bananicultura Atividade & 3 \\
principal & 1 \\
Crédito & 1 \\
Escolaridade & 0 \\
Idade & 0 \\
Mão-de-obra Familiar & 3 \\
Posse & 0 \\
Renda total & 2 \\
Residência & \\
& \\
McFadden R & \\
Estatística LR &
\end{tabular}

Fonte: Dados da pesquisa.

Como citado no método de análise, os coeficientes estimados pelo modelo Probit não medem diretamente a influência das variáveis independentes sobre a variável dependente, sendo necessário, para isso, calcular os seus efeitos marginais. Assim, o efeito marginal indica a variação absoluta na variável dependente, dada por uma variação unitária na variável independente. Embora alguns trabalhos utilizem o efeito marginal para análise com variáveis independentes dummy, calculando-o considerando a variável discreta como se fosse continua (o que não é correto matematicamente) ou, ainda, fazendo a diferença entre a probabilidade quando a variável assumia o valor 1 e quando assumia o valor zero, aqui optou-se por não utilizar tal conceito nesse caso. Isso, pois, de acordo com Soares et al. (2002), a análise de efeito marginal associado a uma variável dummy, como no caso da assistência técnica, não é aplicável, pois essa análise só faz sentido quando a variável explicativa é contínua e não dicotômica. Assim, para essa variável a análise com o efeito marginal não pode ser feita. De igual forma, também não é possível a sua utilização 
para as variáveis Bananicultura como Atividade Principal, Crédito, Escolaridade, Posse e Residência, por serem variáveis discretas.

O sinal do coeficiente da variável Bananicultura como Atividade Principal foi positivo no nível de 5\%, como pode ser visto na Tabela 2 . O resultado ora descrito revela que, quando o produtor tem na bananicultura sua atividade principal, a probabilidade de haver adoção de tecnologia é maior. Isso pode ocorrer em razão da maior atenção dada à bananicultura por esses produtores, em função da maior necessidade de obter bons resultados com a cultura, uma vez que ela é sua principal fonte de renda.

A variável crédito se mostrou significativa no nível de 10\%, com sinal positivo. Resultados semelhantes foram encontrados por Mesquita (1998) e Souza (2000). Em relação a isso, Vicente (1997) ressaltou a importância do crédito rural, afirmando ser ele um fator conjuntural que exerce o papel de promotor da modernização da agricultura.

O nível de escolaridade foi significativo no nível de 10\%. A relação positiva entre a escolaridade e a adoção de tecnologia parece ser consenso entre os autores, que estudaram o assunto. Esse resultado pode ser encontrado também nos trabalhos de Ribeiro (1989), Carbajal (1991), Burton et al. (1998), Holanda Júnior (2000) e Silva e Carvalho (2002). De acordo com CNA (1999), o nível de escolaridade é importante para determinar a capacidade do produtor de se adaptar aos novos cenários do mercado e de decodificar as informações pertinentes a novas tecnologias e práticas de cultivo. Ainda segundo esse mesmo autor, os ajustamentos exigidos pelos mercados geralmente implicam adoção de novos "pacotes" tecnológicos e a escolha correta entre as tecnologias mecânicas, bioquímicas e organizacionais, que dependem do nível de escolaridade e da aptidão para adquirir as informações e adaptá-las às particularidades de cada estabelecimento.

Obteve-se uma relação significativa no nível de 5\% entre a variável idade e a probabilidade de adoção de tecnologia. Segundo Holanda Júnior (2000), a idade pode ter influência na administração da propriedade em 
decorrência da expectativa dos produtores mais jovens, que buscam mudanças e melhoria nos negócios. Porém, de acordo com Carbajal (1991), esse assunto é polêmico e não há consenso entre os resultados dos trabalhos realizados sobre a relação entre idade e nível tecnológico. Esse autor também encontrou relação positiva entre as duas variáveis. De acordo com o efeito marginal calculado, para cada variação de um ano na idade do produtor há um acréscimo de 0,009 na probabilidade de adoção de tecnologia adequada para produção de banana. Como pode ser observado, embora significativa, essa variável tem pequena influência sobre tal probabilidade.

A disponibilidade de mão-de-obra familiar não se mostrou significativa nos níveis de significância utilizados, indicando que a existência de trabalhadores da própria família do produtor não é um fator preponderante na decisão de adotar tecnologia no caso analisado.

A posse da terra apontou significância no nível de 5\%, com sinal positivo. O coeficiente dessa variável foi o mais elevado da equação estimada, evidenciando a sua relevância para a adoção de tecnologia na bananicultura de Mauriti. Souza (2000) encontrou resultado semelhante quanto à relação entre posse da terra e adoção de tecnologia. A influência positiva da posse da terra sobre a probabilidade de adoção de tecnologia aqui encontrada pode ser conseqüência do fato de que, sendo proprietário da terra, torna-se mais fácil para o produtor obter crédito nas instituições financiadoras e, assim, realizar mais investimentos. Além disso, o fato de não pagar o arrendamento da terra estimula o produtor a investir na bananicultura, pois, dessa forma, terá retornos maiores.

A variável Residência foi significativa no nível de 5\%. O sinal positivo do coeficiente estimado indica que o fato de o bananicultor morar na propriedade concorre positivamente para a probabilidade de adoção de tecnologia adequada ou próxima da adequada na produção de banana. $\mathrm{O}$ sinal positivo encontrado pode decorrer do grande número de bananicultores unicamente agricultores, não desenvolvendo outra atividade. Esses produtores dedicam à bananicultura maior tempo, esforço 
e monitoramento de tecnologias, como tratos culturais e irrigação. Como assinalou Holanda Júnior (2000), morar na propriedade contribui para aumentar o tempo de dedicação à atividade agrícola realizada, facilitando a identificação e solução de problemas, tendo como resultado uma melhor administração da propriedade.

A relação entre a probabilidade de adoção de tecnologia e a variável Renda Total foi significativa no nível de $5 \%$, e o coeficiente estimado apresentou sinal positivo. Isso evidencia que bananicultores com renda familiar total mais elevada têm maiores possibilidades de ter maior nível tecnológico. O coeficiente da variável Renda Total indica que, para cada variação de $\mathrm{R} \$ 1.000,00$ na renda familiar mensal (agrícola e nãoagrícola) do produtor, haverá um acréscimo de 0,0132 na probabilidade de adotar tecnologia adequada ou próxima da adequada na produção de banana.

O acerto total do modelo foi de $86,84 \%$, isto é, esse foi o percentual de acerto na classificação de adoção de tecnologia dos produtores de banana de Mauriti, obtido com o modelo estimado. Esse valor apontou que há boa aderência entre o fenômeno estudado e o modelo utilizado.

\section{Conclusões}

As características socioeconômicas dos produtores que têm influência positiva e significativa sobre a probabilidade de adoção de tecnologia são Renda Total, Local da Residência, Assistência Técnica, Posse da Terra, Idade, Bananicultura como Atividade Principal, Escolaridade e Crédito.

Os dados apresentados ao longo do trabalho permitiram concluir que os produtores de banana de Mauriti utilizavam pouco mais de $50 \%$ das tecnologias recomendadas para a cultura. 
A tecnologia adotada na Gestão era insuficiente e as de Colheita e Póscolheita, regulares. As tecnologias de Mudas, Adubação e Tratos Culturais apresentaram um bom nível de adoção e as de Irrigação e de Fitossanidade, ótimo nível de adoção.

Para que a bananicultura de Mauriti e do Agropolo Cariri se expanda e possa alcançar mais mercados, faz-se necessário que haja uma elevação do padrão tecnológico utilizado. Para tanto, sugere-se que seja estimulada a intensificação da adoção de tecnologia, principalmente daquelas adotadas de forma regular e insuficiente (colheita, pós-colheita e gestão), para que o subsetor se torne mais competitivo, produza com maior qualidade e obtenha melhores preços, o que serve como estímulo aos produtores para realização de investimentos. Ainda como um fator de estímulo à adoção de novas tecnologias, deve-se destacar a existência de assistência técnica como forma de identificar e apresentar soluções para problemas que impeçam o desenvolvimento da região.

Convém ressaltar e sugerir políticas que melhorem o nível de escolaridade dos bananicultores de Mauriti, pois tais políticas elevam não somente o patamar de educação formal dos produtores, mas também ampliam seu poder de percepção e aprendizado.

\section{Referências}

BURTON, M. et al. Adoção de tecnologias sustentáveis no Paraná. Revista de Economia e Sociologia Rural, Brasília: SOBER, 1998.

CARBAJAL, A. C. R. Fatores associados à adoção de tecnologias na cultura do caju: um estudo de caso. Fortaleza: UFC, 1991. 122 f. Dissertação (Mestrado em Economia Rural) - Universidade Federal do Ceará, Fortaleza. 
CAVALCANTI, N. de B. et al. Nível tecnológico da agricultura familiar na região semi-árida da Bahia. In: CONGRESSO BRASILEIRO DE ECONOMIA E SOCIOLOGIA RURAL, 36, 1998, Poços de Caldas. Anais ... Poços de Caldas, MG, 1998.

CONFEDERAÇÃO Nacional da Agricultura - CNA. Um perfil do agricultor brasileiro: suas principais tendências e implicações para o treinamento dos pequenos proprietários e trabalhadores rurais. Brasília, 1999. 50 p. (Coletânea Estudos Gleba, 9).

CORDEIRO, Z. J. M. Banana produção: aspectos técnicos. Brasília: EMBRAPA, 2000. 143 p.

ELIAS, D. Globalização e modernização agrícola. Revista Paranaense de Geografia, Paraná, 1996. Disponível em: <ww.agbcuritiba.hpg.com.br>. Acesso em: 20 Out. 2001.

FAVERET FILHO, P.; ORMOND, J. G. P.; PAULA, S. R. L. Fruticultura brasileira: a busca de um modelo exportador. BNDES Setorial, Rio de Janeiro, n. 9, p. 191-226, Mar. 1999.

GREENE, W. H. Econometric analysis. 3.ed. New Jersey: PrenticeHall, 1997.

GUJARATI, D. N. Econometria básica. 3. ed. São Paulo: Makron Books, 2000. 846 p.

HOLANDA JÚNIOR, F. I. F. de. Análise técnico-econômica da pecuária leiteira no Município de Quixeramobim - Estado do Ceará. Fortaleza: UFC/CCA/DEA, 2000. 103 f. Dissertação (Mestrado em Economia Rural). - Universidade Federal do Ceará, Fortaleza.

LIMA, R. C. Modelos de resposta binárias: especificação, estimação e inferência. Revista Agricultura em São Paulo, v. 43, 1996. 
MADALLA, G. S. Introduction to econometrics. 2. ed. New York: Mcmillan, 1992. $631 \mathrm{p}$.

MESQUITA, T. C. Estudos de economia agrícola. Sobral, RN: Edições UVA, 1998.

MIRANDA, E. A. de A. Inovações tecnológicas na viticultura do sub-médio São Francisco. Recife: UFPE, 2001. 199 f. Tese (Doutorado em Economia) - Universidade Federal de Pernambuco, Recife.

RIBEIRO, D. G. L. Adoção de tecnologia na agricultura de canade-açúcar - Microrregião do Cariri-CE. Fortaleza: UFC/CCA/DEA, 1989. 92 f. Dissertação (Mestrado em Economia Rural) - Universidade Federal do Ceará, Fortaleza.

SANTOS, M. Fatores sócio-econômicos relacionados com adotabilidade de práticas agropecuárias no Estado de Minas Gerais. Viçosa, MG: UFV, 1997. 142 f. Dissertação (Mestrado em Economia Rural) - Universidade Federal de Viçosa, Viçosa.

SANTOS, S. M. dos. A análise dos determinantes em capacitação tecnológica nas empresas brasileira: evidências empíricas. Fortaleza: CAEN-UFC, 2000. (Estudos Económicos-CAEN).

SILVA, C. R. L. da; CARVALHO, M. A. de. Uma análise dos fatores que determinam a adoção de tecnologia: aplicação de um modelo de dados de contagem nas regiões de Ourinhos e Ribeirão Preto, São Paulo. In: CONGRESSO BRASILEIRO DE ECONOMIA E SOCIOLOGIA RURAL, 40., 2002, Passo Fundo. Anais ... Passo Fundo, RS: 2002. CDROM.

SOARES, F. de A. et al. Reuso de água industrial: o caso das empresas do distrito industrial de Maracanaú - Ceará. In: CONGRESSO BRASILEIRO DE ECONOMIA E SOCIOLOGIA RURAL, 40., 2002, Passo Fundo. Anais ... Passo Fundo, RS, 2002. CD-ROM. 
SOUZA, A. T. Aspectos econômicos da cultura da banana. Disponível em: 〈www.icepa.com.br/agroindicadores/opinião/analise_banana.htm>. Acesso em: 13 Maio 2002.

SOUZA, A. T. Banana - síntese anual da agricultura de Santa Catarina. Florianópolis: ICEPA, 2002.

SOUZA, F. L. Estudo sobre o nível tecnológico da agricultura familiar no Ceará. FC/CCA/DEA, Fortaleza. 2000. 107 f. Dissertação (Mestrado em Economia Rural) - Universidade Federal do Ceará, Fortaleza.

VICENTE, J. R. Determinação da adoção de tecnologia na agricultura paulista. In: CONGRESSO BRASILEIRO DE ECONOMIA E SOCIOLOGIA RURAL, 35., 1997, Natal. Anais... Natal, 1997. Comunicações - CD-ROM.

\begin{abstract}
Utilization of technology in agriculture makes it productive and promissing, contributing to its development. Investiments done by Govern of Ceará in agriculture have concentrate mainly on irrigated agriculture, through the agropoles, prevailing fruitgrowing, banana being one of the chief crops. Banana crop performs an important role for cearense agriculture, however, it is generally characterized by low productivity, low technological level and large losses in production. The objective of this study was to measure the technological level of the irrigated banana crop in Agropole Cariri and to verify which are the factors conditioning the technological adoption. The calculated index showed that the technological index adopted in banana crop is classified as good. Technologies of irrigation and phytosanity have adoption level classified as optimum and the ones of the seedlings, adubation and cultural traits as good. Harvest and postharvest technologies levels are classified as regular and the ones of management as insufficient. Socioeconomic variables such as technical assistence, banana crop as main activity, credit, scholarship, age, land owning, income, and place of dwelling contribute positively to increase the probability of producer to adopt the adequate technology in banana crop.
\end{abstract}

Keywords: Technology adoption, banana crop, Agropole Cariri. 